\title{
An overlooked cause of longitudinally extensive spinal cord lesions: Primary central nervous system lymphoma
}

\section{Meng Wang}

Jilin University First Hospital

\section{Baochang Qi}

Jilin University First Hospital

Jinming Han

Jilin University First Hospital

Chunjie Guo

Jilin University First Hospital

Limei Qu

Jilin University First Hospital

Jie Zhu

Karolinska Institutet Department of Neurobiology Care Sciences and Society

tao jin ( $\nabla$ drtao.jin@hotmail.com )

Jilin University First Hospital https://orcid.org/0000-0002-7028-3419

\section{Case Report}

Keywords: Primary central nervous system lymphoma, spinal cord, Longitudinally extensive transverse myelitis, MRI, 18F-FDG-PET/CT

Posted Date: February 12th, 2020

DOI: https://doi.org/10.21203/rs.2.23382/v1

License: (a) (i) This work is licensed under a Creative Commons Attribution 4.0 International License. Read Full License 


\section{Abstract}

Background: Primary central nervous system lymphoma (PCNSL) is a rare and aggressive malignant tumor. It is easy to be misdiagnosed due to its low incidence and unspecific presentations in clinical practice. PCNSL mainly occurs intracranially in the brain while spinal cord is rarely involved.

Case presentation: Here we report a 76-year-old woman who had a suspicious tumor history and presented retardant paralysis, bladder dysfunction and sensory loss of the lower limbs. Magnetic resonance imaging (MRI) of the thoracic spine disclosed longitudinally extensive lesions extending from thoracic 4 (T4) to lumbar 1 (L1) vertebral level with an enhanced nodular lesion noting at levels of T10 and T11 . In order to further identify the cause, the whole body 18 F-fluorodeoxyglucose ( 18 F-FDG) positron emission tomography (PET)/computed tomography (CT) was performed and showed a hypermetabolic nodule corresponded to MRI enhancing lesions, which further suggesting the possibility of a tumor. The patient then underwent a surgical resection and spinal cord biopsy confirmed the diagnosis of non-Hodgkin's lymphoma (diffuse large B-cell type). The patient then received a high-dose chemotherapy based on methotrexate combined with Rituximab. Unfortunately, the symptoms of this patient have not been improved significantly after three rounds of chemotherapy.

Conclusion: Our case indicates that PCNSL may also serve as a possible cause for longitudinally extensive spinal cord lesions, especially the patients who had a suspicious tumor history, MRI enhancing lesion $s$ in the spinal cord corresponded to hypermetabolic nodules on 18 F-FDG-PET/CT at the same level.

\section{Background}

Longitudinally extensive transverse myelitis (LETM) is usually defined as spinal cord lesions that extended vertically through three or more vertebral segments (1). LETM can be usually noted in inflammatory demyelinating diseases in the central nervous system (CNS), such as neuromyelitis optica spectrum disorders (NMOSD), myelin oligodendrocyte glycoprotein (MOG)-antibody disease, multiple sclerosis (MS), acute disseminated encephalomyelitis (ADEM), systemic inflammatory diseases and spinal malignancies (2). LETM caused by PCNSL is rare in clinical practice. The magnetic resonance imaging (MRI) findings are important to distinguish between PCNSL and other myelopathic etiologies. Furthermore, the combination of MRI and whole body ${ }^{18} \mathrm{~F}$-fluorodeoxyglucose $\left({ }^{18} \mathrm{~F}-\mathrm{FDG}\right)$ positron emission tomography (PET)/computed tomography (CT) makes the diagnosis more convincing. Researchers have showed that the specificity and sensitivity of ${ }^{18} \mathrm{~F}-\mathrm{FDG}$-PET/CT in the diagnosis of PCNSL are 0.86 and 0.88 respectively (3). Here we report a patient with pathologically confirmed spinal cord PCNSL. Our case highlights that PCNSL in the spinal cord may also serve as a possible cause for longitudinally extensive spinal cord lesions.

\section{Case Presentation}


A 76-year-old Chinese woman was admitted to the Department of Neurology and Neuroscience Center in the First Hospital of Jilin University, Changchun, China. She had been healthy until five months earlier when progressive weakness of her left lower limb was noticed. She reported no significant changes of her body weight recently. Brain MRI demonstrated lesions in the right temporal and occipital lobes. The lesions showed hypointense signal on T1-weighted images, hyperintense signal on T2-weighted images and fluid attenuated inversion recovery (FLAIR) sequence (Fig. 1: ABC). A clinical diagnosis of tumor was originally considered by the local hospital and the patient was therefore received radiotherapy. One week after treatment, her strength of lower limbs started to improve. Two months later after radiotherapy, repeated brain MRI revealed the lesions were ameliorated compared with the initial scans (Fig. 1: DEF). She was admitted to our hospital due to acute onset of lower limbs weakness and bladder dysfunction that quickly culminated within 2 days. Physical examinations reflected the features of the spinal cord shock stage, concretely showing retardant paralysis and anesthesia below T10. Deep tendon reflexes were disappeared in both lower limbs, while normal in the upper extremities. Bilateral Babinski signs were negative. Urinary catheter was used due to urinary retention. Other physical examination results were unremarkable.

Routine laboratory investigations such as C-reactive protein, complete blood count, blood biochemical analysis, Vitamin B12 level, human immunodeficiency virus (HIV), syphilis and circulating tumor markers were normal. Serological assays for autoimmune diseases and connective tissue diseases such as thyroid status, antinuclear antibodies were also negative. Aquaporin-4 (AQP4) antibody, MOG-antibody and paraneoplastic antibodies including CV2/collapsin response mediator protein5 (CRMP5), Ma2, RI, Yo, $\mathrm{Hu}$ and Amphiphys in both serum and CSF were negative. CSF analysis showed $36 \times 10^{6} / \mathrm{L}$ of cell number, $0.67 \mathrm{~g} / \mathrm{L}$ of protein and no oligoclonal bands. Brain MRI showed lesions in the right parietal lobe (Fig. 2: $A B C$ ). The lesions were nodular enhancement on T1-weighted contrast enhanced images (Fig. 2: D). MRI of the spinal cord showed a longitudinally extensive hypointensive lesion from thoracic 4 (T4) to lumbar 1 (L1) vertebral level on T1-weighted images, hyperintensive signal on T2-weighted images and T2 fat-suppressed images, respectively (Fig. 3: ABC). Axial thoracic MRI images showed a mass lesion located in the ventral part of the cord (Fig. 3: E). Post contrast-enhanced MRI revealed an enhanced nodular lesion (Fig. 3: DF). In support of MRI results, ${ }^{18}$ F-FDG-PET/CT demonstrated hypermetabolism lesions (Fig. 3: GH). Then, the patient received a tumor resection 8 days after admission. Spinal cord biopsy was performed at around T10-T11 level, confirming the diagnosis of diffuse large B cell lymphoma. Hematoxylin and eosin-staining staining showed diffuse proliferation of large atypical lymphocytes. Immunohistochemical studies demonstrated that neoplastic lymphocytes are positive for B-cell lymphoma-2 (Bcl-2), CD20 and c-Myc (Fig. 4). Thus, the patient received high-dose methotrexate (HD-MTX) combined with Rituximab chemotherapy. The patient discharged from the hospital after the first chemotherapy. The patient was followed up by phone. Unfortunately, the patient could not tolerate high-dose chemotherapy drugs after receiving three rounds of chemotherapy, therefore she decided to forgo her fourth chemotherapy. Until now, the symptoms of flaccid paralysis, sensory disorder and persistent bladder dysfunction did not alleviate significantly during four months follow-up period. 


\section{Discussion}

PCNSL represents $0.8-6.6 \%$ of all CNS neoplasms and only $1 \%$ of all lymphoma diagnoses (4). Intramedullary lymphoma with spinal cord involvement is rare, accounting for $1 \%$ of PCNSL (5). The average age of the disease was 50 years, with a higher prevalence being observed in men and 5060 years being the peak period of incidence (6). The clinical symptoms of primary intramedullary spinal cord lymphoma are usually non-specific and related to the levels of the spinal cord involvement (7). Clinically, the onset symptoms were subacute or insidious and then became progressive in nature. In this case, the patient was an old woman who presented with acute onset sensory and motor impairments that quickly culminated within 2 days, which was similar to clinical features of inflammatory demyelinating disease.

More than half of PCNSL cases were involved the conus medullaris and/or cauda equina (8). The expansile spinal cord is often presented in MRI with isointense to hypointense on T1-weighted images and signal intensity on T2-weighted images, meanwhile they are usually solitary lesions (9-10). In most cases, the lesions were enhanced homogeneously (11). The application of ${ }^{18} \mathrm{~F}-\mathrm{FDG}$-PET/CT is widely used in clinic now. Flanagan et al. reported that spinal cord ${ }^{18}$ F-FDG-PET/CT hypermetabolism in patients with active myelopathy was much more common than inflammatory myelopathies (12). PCNSL has a strong uptake of ${ }^{18} \mathrm{~F}-\mathrm{FDG}$, while the ${ }^{18} \mathrm{~F}$-FDG uptake is not obvious in non-enhancing lesions on MRI (13). Some researchers demonstrated that higher ${ }^{18} \mathrm{~F}$-FDG-PET/CT hypermetabolism and maximum standardized uptake value in PCNSL than other tumors and inflammatory diseases (14-15). Furthermore, the CSF and serum tests are usually inconclusive. In our case, the patient presented with a LETM extending from T4 to L1 level. The lesions displayed hypointensity in T1-weighted images and hyperintensity in T2-weighted images which consistent with other studies (16). Our patient had one enhancing lesion with homogeneous enhancement on imagings with ${ }^{18} \mathrm{~F}-\mathrm{FDG}$-PET/CT showing a hypermetabolic nodule at the level of T10 to T11.

PCNSL is sensitive to chemotherapy and radiotherapy (17). The most effective treatment for newly diagnosed PCNSL is HD-MTX based combination chemotherapy (18). The role of surgery in PCNSL is limited given the characteristic of diffusely infiltrating growth of PCNSL (19). In our case, the risk of radiation therapy-related neurotoxicity is higher in order patients than other young patients.

In conclusion, the reason of LETM is broad. Our case highlights that PCNSL may also serve as a possible cause for longitudinally extensive spinal cord lesions. Therefore, PCNSL in the spinal cord can not be ignored by neurologist. A suspicious tumor history, enhancing MRI lesions in the spinal cord and hypermetabolic nodules on ${ }^{18} \mathrm{~F}$-FDG-PET/CT corresponding to MRI lesions may highly suggestive of spinal cord PCNSL.

\section{Abbreviations}

PCNSL 
Primary central nervous system lymphoma

MRI

Magnetic resonance imaging

${ }^{18}$ F-FDG-PET/CT

${ }^{18} \mathrm{~F}$-fluorodeoxyglucose-positron emission tomography/computed tomography

LETM

Longitudinally extensive transverse myelitis

CNS

Central nervous system

NMOSD

Neuromyelitis optica spectrum disorders

MOG

Myelin oligodendrocyte glycoprotein

MS

Multiple sclerosis

ADEM

Acute disseminated encephalomyelitis

$T$

thoracic

L

lumbar

FLAIR

fluid attenuated inversion recovery

HIV

human immunodeficiency virus

AQP4

Aquaporin-4

CRMP5

collapsin response mediator protein 5

HD-MTX

high-dose methotrexate

\section{Declarations}

\section{Acknowledgements}

Not applicable.

\section{Funding}


This work was supported by grants from the General Program of the National Natural Science Foundation of China (No. 81671177), Young Scholars Program of the National Nature Science Foundation of China (No. 81600923), Natural Science Foundation of Jilin Province Science and Technology Development Plan Project (20190201043JC), the Technology Innovation Program of Jilin Provincial Health and Family Planning Commission of China (No. 2016J040), as well as the grants from the Swedish Research Council (No. 2015-03005) and grants from the First hospital, Jilin University of China.

\section{Authors' contributions}

WM and QB collected the data and drafted the manuscript. HJ performed critical revisions and provided insightful ideas. GC helped to prepare the figures and discussed MRI results. QL helped to discussed biopsy results. ZJ and JT supervised the study.

\section{Ethics declarations}

\section{Ethics approval and consent to participate}

Not applicable

\section{Consent for Publication}

The relatives of this patient provided written informed consent to the publication of the information and images related to this case report.

\section{Availability of data and materials}

Not applicable.

\section{Competing interests}

As this is a case report without experimental intervention, no formal research ethics approval is required. The authors have no conflicts of interest to declare.

\section{References}

1. Sato Douglas Kazutoshi, Misu Tatsuro, Rocha Cristiane Franklin et al. Aquaporin-4 antibody-positive myelitis initially biopsied for suspected spinal cord tumors: diagnostic considerations. Mult. Scler., 2014,20:621-6.

2. Cattan Samuel, Papeix Caroline, Grabli David et al. Early radiological features of severe longitudinally extensive transverse myelitis over time. J. Neurol. Sci., 2019,400:7-9.

3. Zou Y, Tong J, Leng $\mathrm{H}$, et al. Diagnostic value of using 18F-FDG PET and PET/CT in immunocompetent patients with primary central nervous system lymphoma: A systematic review 
and meta-analysis. Oncotarget, 2017, 8(25):41518-41528.

4. Guzzetta Melissa, Drexler Steven, Buonocore Brian et al. Primary CNS T-cell lymphoma of the spinal cord: case report and literature review. Lab Med, 2015,46:159-63.

5. Hochberg F H, Miller D C. Primary central nervous system lymphoma. Journal of Neurosurgery, 1988, 68(6):835-853.

6. Yang Wuyang, Garzon-Muvdi Tomas, Braileanu Maria et al. Primary intramedullary spinal cord Iymphoma: a population-based study. Neuro-oncology, 2017,19:414-421.

7. Han Catherine H, Batchelor Tracy T. Primary Central Nervous System Lymphoma. Continuum (Minneap Minn), 2017,23:1601-1618.

8. Sivri Mesut, Erdoğan Hasan, Allahverdiyev Ilgar et al. A rare cause of spinal mass: primary intramedullary spinal cord lymphoma. Spine J, 2015,15:e43-4.

9. Hazenfield John Michael, Gaskill-Shipley Mary F, Neoplastic and Paraneoplastic Involvement of the Spinal Cord. Semin. Ultrasound CT MR, 2016,37:482-97.

10. Nakamizo Tomoki, Inoue Haruhisa, Udaka Fukashi et al. Magnetic resonance imaging of primary spinal intramedullary lymphoma. J Neuroimaging, 2002,12:183-6.

11. Sutherland Tom, Yap Kelvin, Liew Elaine et al. Primary central nervous system lymphoma in immunocompetent patients: a retrospective review of MRI features. J Med Imaging Radiat Oncol, 2012,56:295-301.

12. Flanagan Eoin $P$, Hunt Christopher $H$, Lowe Val et al. [(18)F]-fluorodeoxyglucose-positron emission tomography in patients with active myelopathy. Mayo Clin. Proc., 2013, 88: 1204-12.

13. Chiavazza C, Pellerino A, Ferrio F, et al. Primary CNS Lymphomas: Challenges in Diagnosis and Monitoring. BioMed Research International, 2018, 2018:1-16.

14. Ayrignac, Xavier, Orgeval, Jessica, Mariano-Goulart, Denis. Sensitivity of [18F]-FluorodeoxyglucosePositron Emission Tomography in Patients With Active Myelopathy. Mayo Clinic Proceedings, 2014.

15. Biswas Shamick,Nagaraj Chandana,Mangalore Sandhya et al. Tumefactive Demyelination versus Primary Central Nervous System Lymphoma on F-Fluorodeoxyglucose Positron Emission Tomography Magnetic Resonance Imaging: A Twist in the Tale. Indian J Nucl Med, 2019, 34: 237240.

16. Teo Mario K,Mathieson Calan,Carruthers Ross et al. Cauda equina lymphoma--a rare presentation of primary central nervous system lymphoma: case report and literature review. Br J Neurosurg, 2012, 26: 868-71.

17. Han Catherine H, Batchelor Tracy T, Diagnosis and management of primary central nervous system lymphoma. Cancer, 2017, 123: 4314-4324.

18. Löw Sarah, Han Catherine H, Batchelor Tracy T. Primary central nervous system lymphoma. Ther Adv Neurol Disord, 2018, 11: 1756286418793562.

19. Grommes Christian, DeAngelis Lisa M. Primary CNS Lymphoma. J. Clin. Oncol., 2017, 35: $2410-2418$. 


\section{Figures}
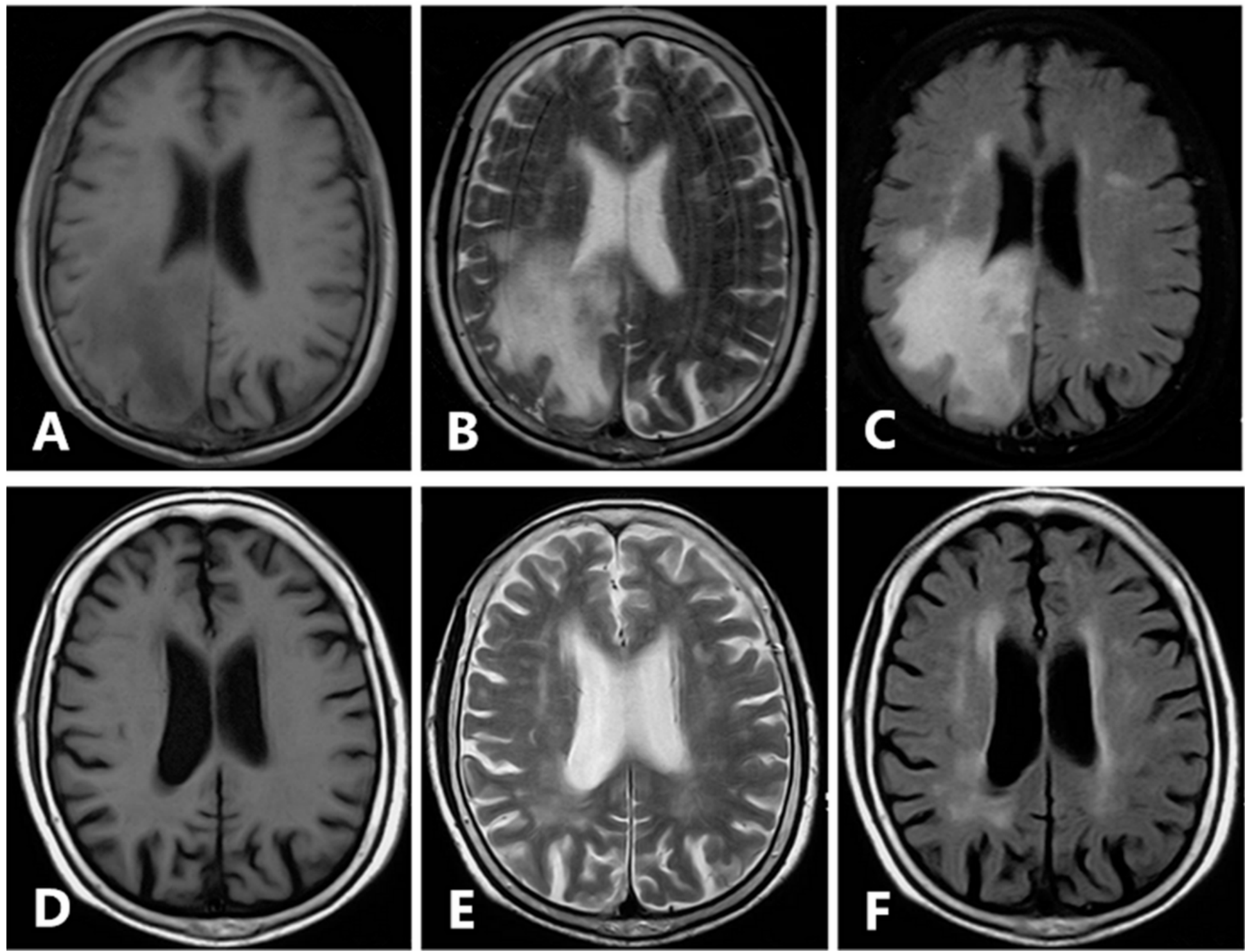

Figure 1

Initial brain MRI before radiotherapy showed lesions in the right temporal and occipital lobes. The lesions showed hypointense signal on T1-weighted images (A), hyperintense signal on T2-weighted (B) and FLAIR images (C). Repeat MRI showed the brain lesions have almost been disappeared (DEF). 

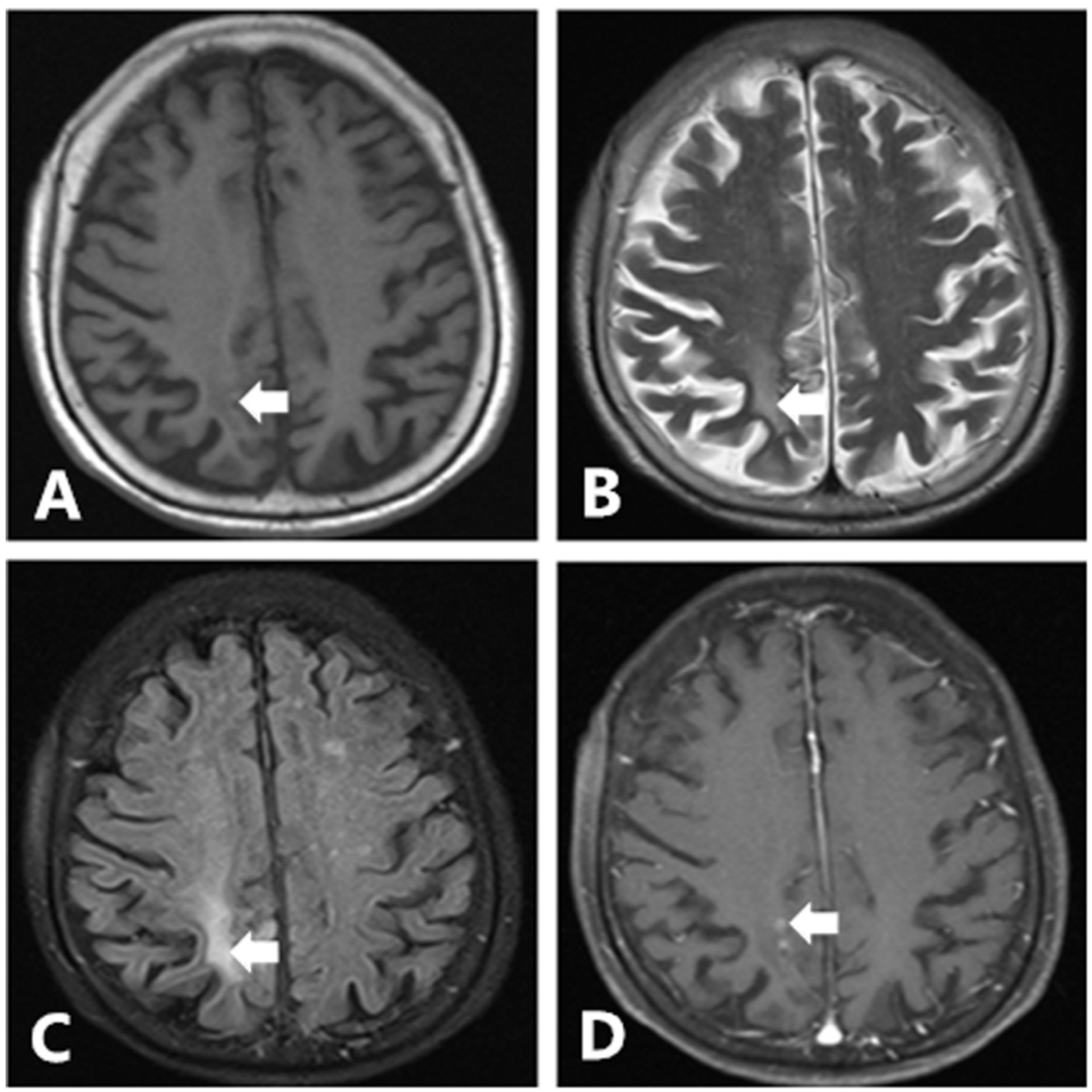

\section{Figure 2}

Brain MRI showed lesions in the right parietal lobe. The lesions were hypointense on T1-weighted images (A), hyperintense on T2-weighted (B) and FLAIR images (C) (white arrows). (D) T1-weighted contrast enhanced images reveled enhancing nodular lesions in the right parietal lobe (white arrows). 

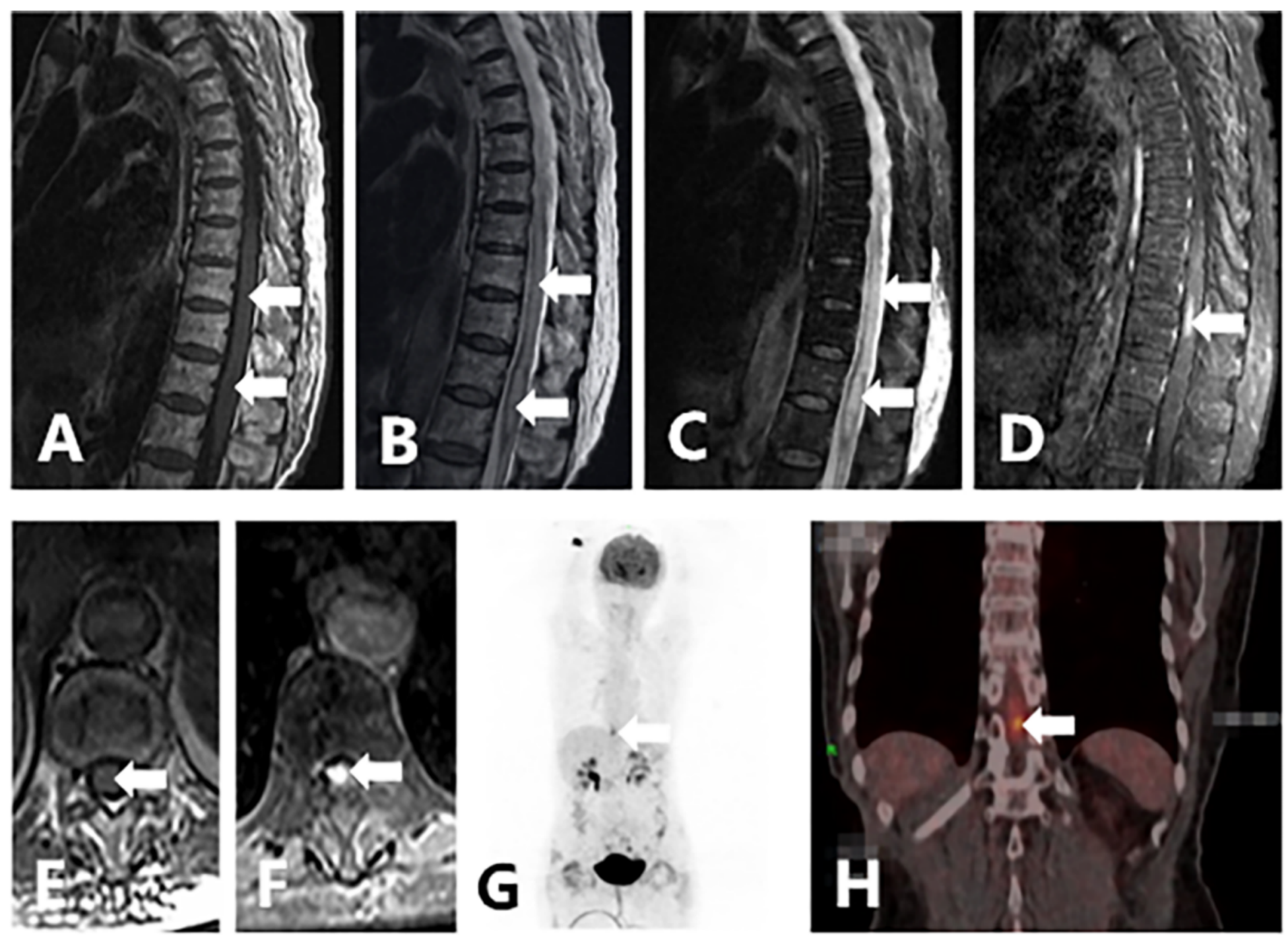

Figure 3

MRI of the thoracic cord and whole body 18F-FDG-PET/CT. The T1-weighted (A), T2-weighted (B) and T2 fat-suppressed images(C) demonstrated a longitudinally extensive hypointensive lesion from thoracic 4 (T4) to lumbar 1 (L1) vertebral level (white arrows). (D) Sagittal MRI of thoracic spine revealed an enhanced nodular lesion at T10 to T11 level (white arrows). (EF) Axial thoracic MRI images showed a mass lesion located in the ventral part of the cord (white arrows). (GH) 18F-fluorodeoxyglucose positron emission tomography/computed tomography (18F-FDG-PET/CT) showed no other primary lesions. 

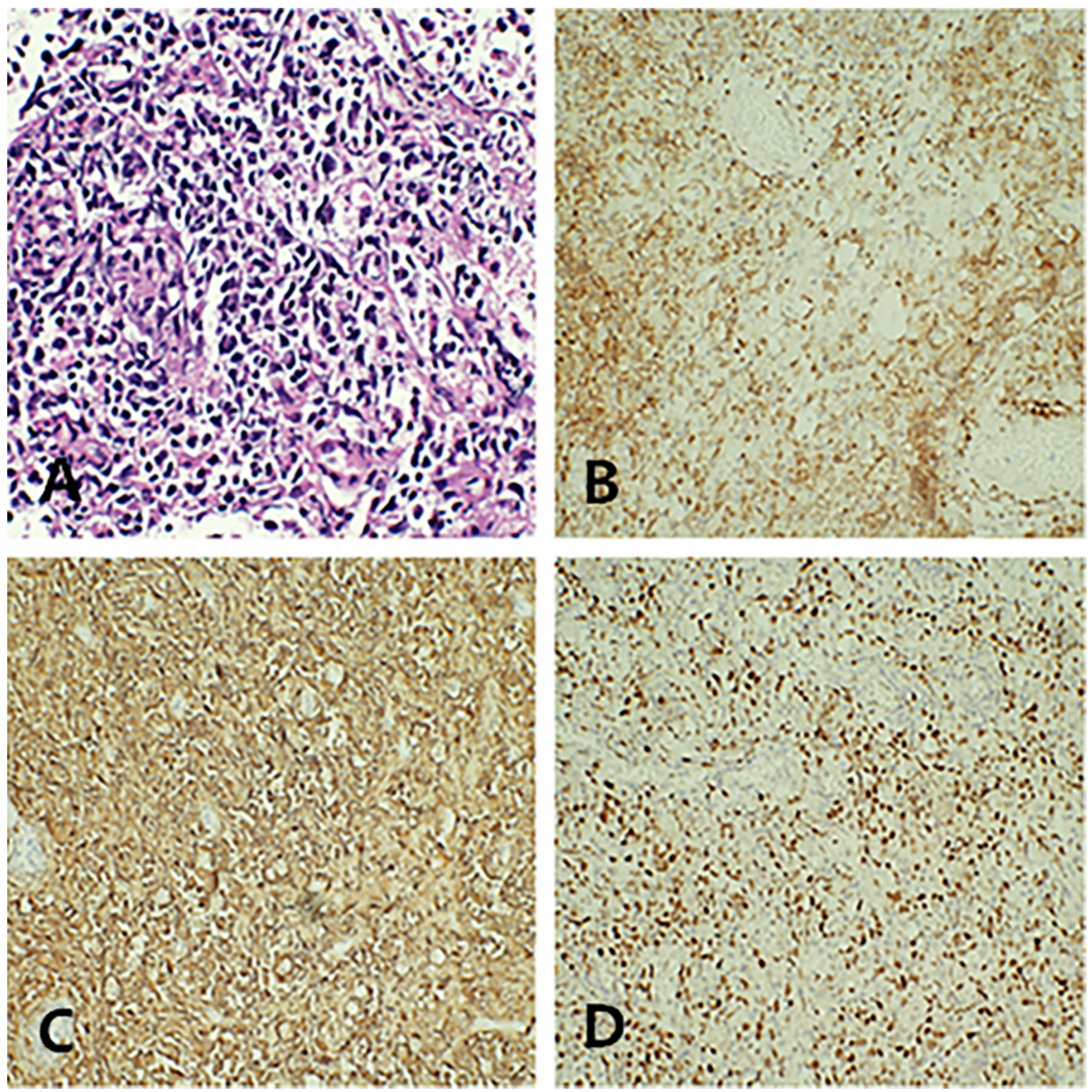

Figure 4

Histopathology of the biopsy. (A) A pathological specimen shows diffuse proliferation of large atypical lymphocytes (A, hematoxylin and eosin stain, $\times 40)$. (B) Immunohistochemical studies show that neoplastic lymphocytes are positive for Bcl-2 (B: immunohistochemical staining for Bcl-2), CD20 (C: immunohistochemical staining for CD20) and c-Myc (D: immunohistochemical staining for c-Myc). 
This is a list of supplementary files associated with this preprint. Click to download.

- CAREchecklist.pdf 